\title{
Stapes surgery in patients with profound hearing loss secondary to otosclerosis
}

\author{
L Dwyer-Hemmings, J G Manjaly, R Nash, A K Mukherjee, J A Lavy \\ Royal National Throat, Nose and Ear Hospital, UK
}

\begin{abstract}
Objective To evaluate the audiometric and quality of life benefit of stapes surgery in patients with otosclerosis and profound hearing loss, who would meet audiometric criteria for cochlear implantation (the National Institute for Clinical Excellence recommends cochlear implants for bilateral air and bone conduction hearing thresholds greater than $90 \mathrm{~dB}$ at 2 and $4 \mathrm{kHz}$ without using a hearing aid).
\end{abstract}

Study Design Retrospective study and questionnaire

Setting Tertiary care hospital

Patients 33 adult patients (35 ears) who met criteria for cochlear implantation. Mean age 63.6 (range 40 to 85 ).

Intervention Stapedotomy with prosthetic implantation

Main outcome measures Pure tone hearing threshold averages (PTA) were recorded at $0.5 \mathrm{kHz}, 1 \mathrm{kHz}, 2 \mathrm{kHZ}$ and $3 \mathrm{kHz}$, before and after surgery. Thresholds at 2 and $4 \mathrm{kHz}$ were compared separately. Glasgow Benefit Inventory (GBI) was used in 20 patients to assess quality of life changes. GBI is a validated person-centred quality of life questionnaire that assesses the impact of the operation on social, physical and general domains. 18 questions are scored, averaged, and normalised so that -100 indicates entirely negative outcomes and +100 indicates overwhelmingly positive outcomes. A high questionnaire response rate of $77 \%$ supports the accuracy of our data.

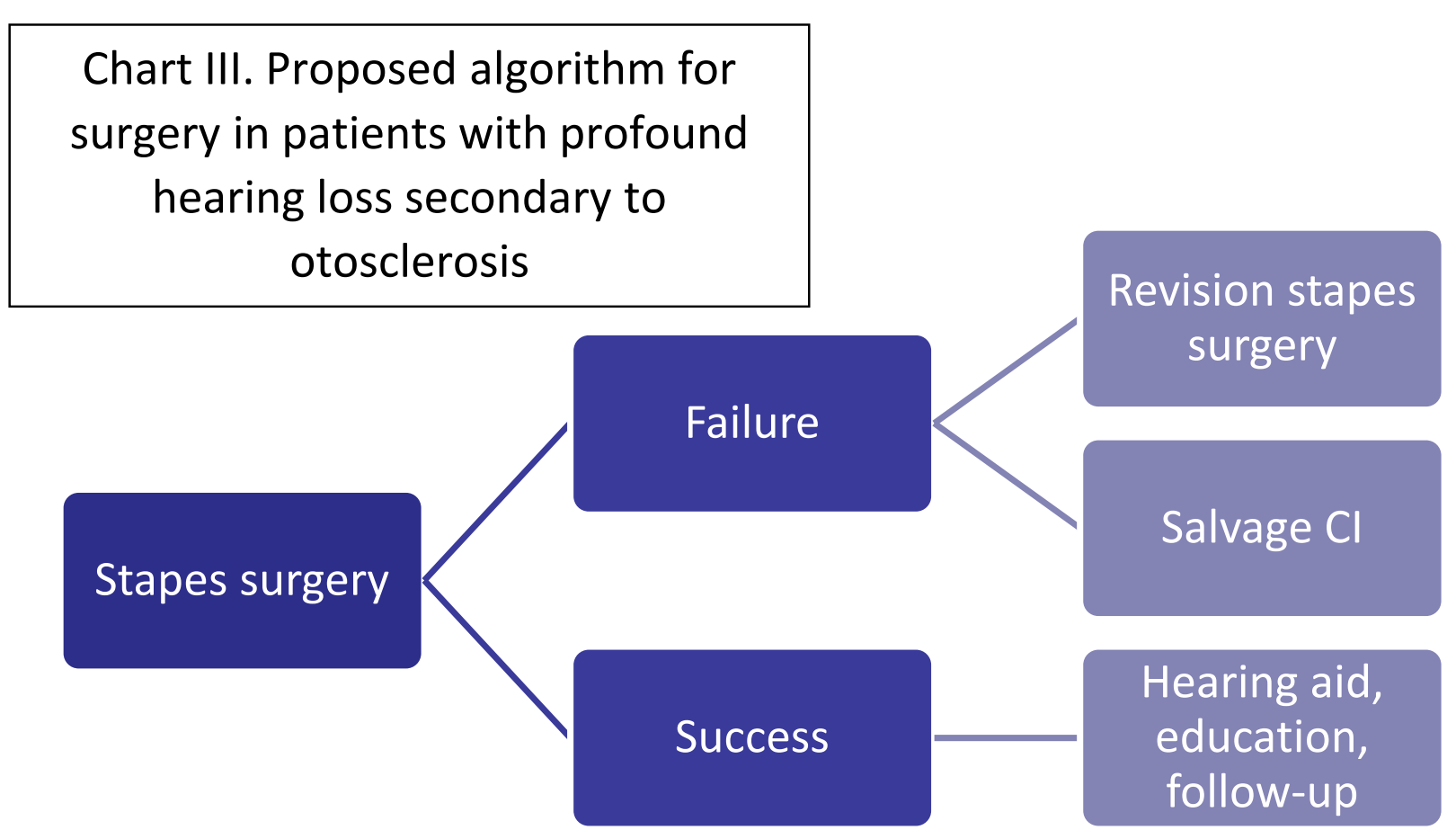

NICE. Cochlear Implantation for children and adults with severe to profound deafness (TA166). 2009.

Robinson et al. "Measuring Patient Benefit From

Otorhinolaryngological Surgery." 1996;105 (6): 415-422. Ann

Otol Rhinol Laryngol

Calmels et al. "Very Far-Advanced Otosclerosis." Acta

Otolaryngol 2007;127 (6): 574-578. 18.

Berrettini et al. "Far advanced otosclerosis." J Laryngol Otol 2004;33 (3):165-171.

Van Loon et al. "Stapedotomy in cochlear implant

candidates." Otol Neurotol 2014;35 (10): 1707-1714.

Abdurehim et al. "Stapedotomy Vs Cochlear Implantation."

Otolaryngol Head Neck Surg 2016;155 (5): 764-770.

Kabbara et al. "Decisive Criteria Between Stapedotomy And

Cochlear Implantation." Otol Neurotol 2015;36 (3): e73-e78.
Results $80 \%$ of ears demonstrated improvement in air conduction thresholds. Mean threshold improvement postsurgery was $26.3 \mathrm{~dB}$. 4 patients experienced unchanged air conduction hearing thresholds, while 3 patients experienced worsened thresholds. Mean GBI score was +17 . Hearing aid use decreased in $25 \%$ and stopped entirely in $25 \%$.

Conclusion Our study shows that stapes surgery in patients with profound hearing loss secondary to otosclerosis can produce significant improvements in air conduction hearing thresholds, decrease reliance on hearing aids, and increase quality of life as measured by GBI. This leads us to agree with the several other authors who have suggested that stapes surgery should be used in the first instance as surgical management for this patient group (chart 3). ${ }^{71821109}$ Meeting criteria for an intervention does not necessitate the use of that intervention, and due to the high chance of a positive outcome, stapes surgery should be offered first, with cochlear implantation reserved for patients who show an unsatisfactory response.

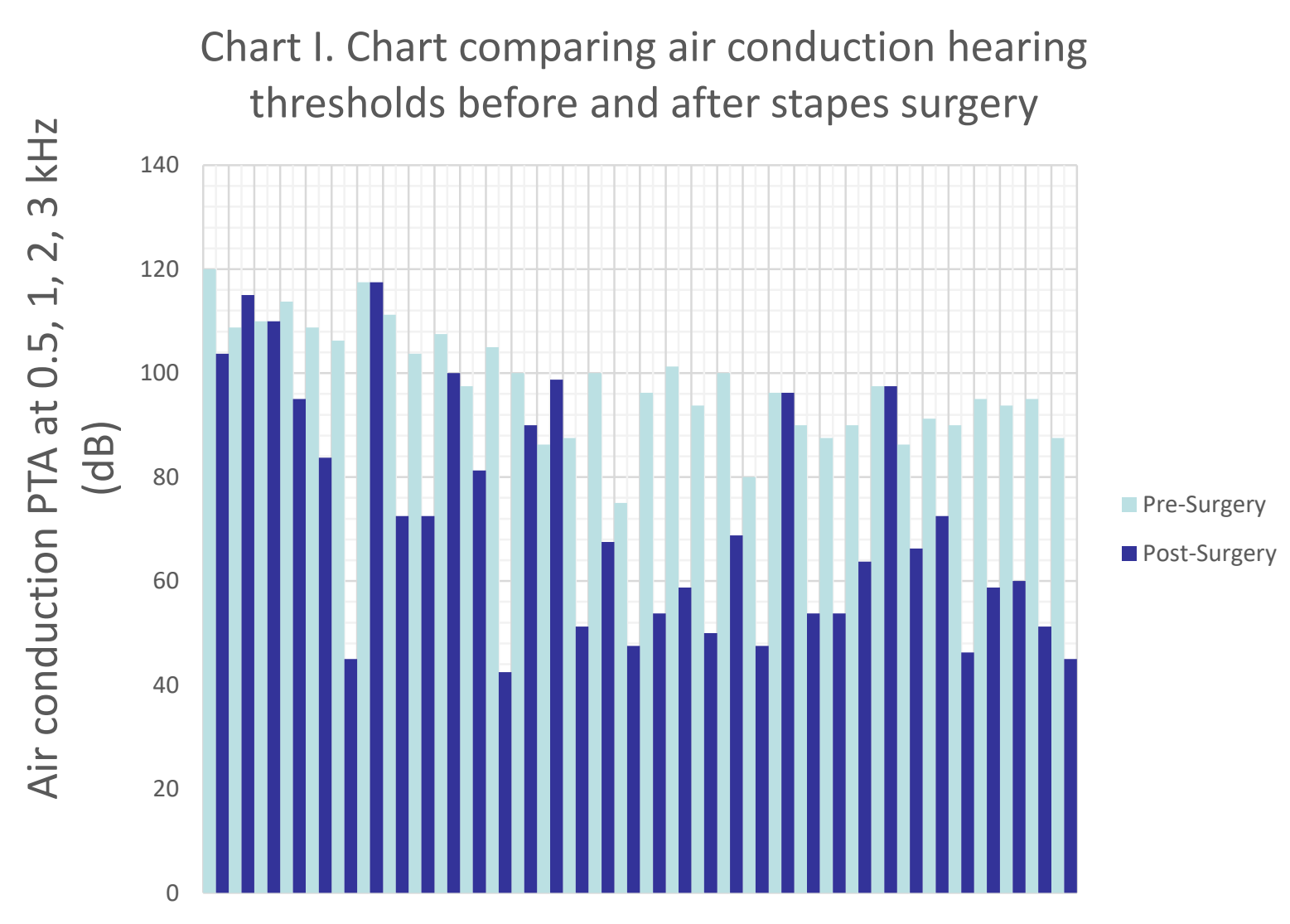

Chart II. Scatter chart showing average GBI against air conduction hearing threshold improvement

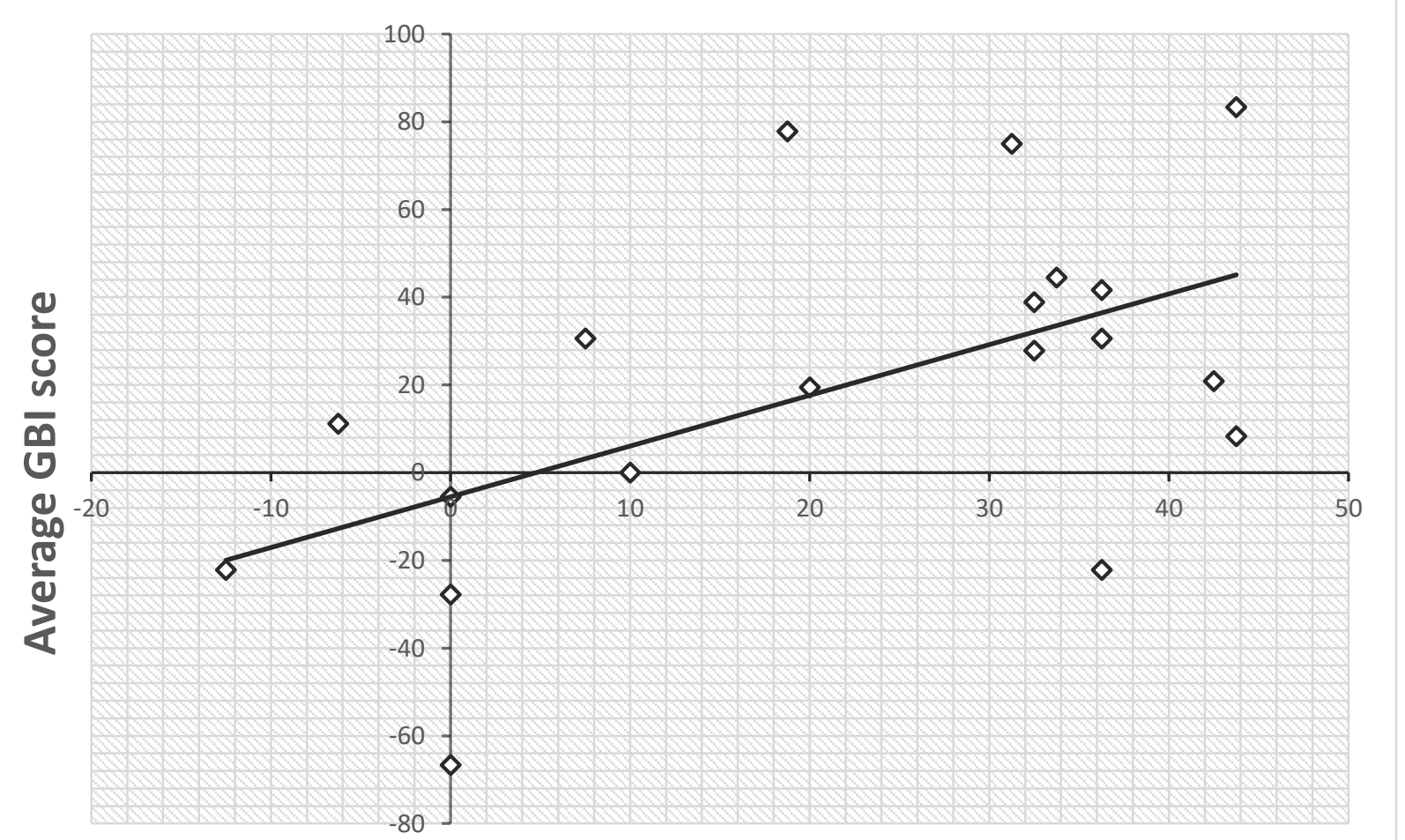

Air conduction hearing threshold PTA at $0.5,1,2,3 \mathrm{kHz}$ improvement (dB)

Copyright @ 2018 The Authors. Correspondence to L Dwyer-Hemmings, UCL Medical School, 74 Huntley Street, London WC1E 6BT, UK. Email: Itdwyerhemmings@gmail.com 